\title{
Conflicts as Aversive Signals for Control Adaptation
}

\section{Gesine Dreisbach ${ }^{1}$ and Rico Fischer ${ }^{2}$}

@SAGE

${ }^{1}$ Department of Experimental Psychology, University of Regensburg, and ${ }^{2}$ Department of Psychology,

Technische Universität Dresden

\begin{abstract}
The dynamic adaptation of cognitive control in the face of competition from conflicting response tendencies is one of the hallmarks of flexible human action control. Here, we suggest an alternative framework that places conflicttriggered control adaptation into the broader context of affect regulation. Specifically, we review evidence showing that (a) conflicts are inherently aversive, that (b) aversive stimuli in the absence of conflict also trigger behavioral adjustments, and, finally, that (c) conflict stimuli do trigger processes of affective counter-regulation. Together with recent findings showing that conflict-triggered control adaptation depends on the subjective experience of the conflict, we suggest that it is the subjective aversive conflict experience that originally motivates control adaptations. Such a view offers new perspectives for investigating and understanding intra- and interindividual differences in the regulation of cognitive control by differentiating between the individual sensitivity to experience and the individual ability to utilize the aversive signal.
\end{abstract}

\section{Keywords}

response conflict, conflict monitoring, aversive signal, cognitive control, affective counter-regulation

Cognitive control refers to the unique ability to carry out a weak but intended response in the face of competition from a dominant habitual response (cf. Miller \& Cohen, 2001). For example, when booting up the computer to work on an overdue review, you might experience the temptation to open your e-mail client instead of your word-processing program. In order to deal with such response conflicts, the cognitive system has developed a monitoring system that detects conflicts (i.e., the concurrent activation of conflicting response tendencies) in the ongoing processing stream, and it uses this information to increase cognitive control (e.g., Botvinick, Braver, Barch, Carter, \& Cohen, 2001). This so-called sequential control adaptation has been subject to tremendous research in the last decade, the basic finding being that in classical interference tasks, interference caused by response conflict in one trial is substantially reduced, if not eliminated, in the subsequent trial (for a review, see Egner, 2007). Conflict adaptation can thus be considered a prime example of flexible human action control. Here, we aim to provide an answer to the question of what exactly it is about the conflict that initially motivates this control adaptation.

\section{Sequential Control Adaptation: The Gratton Effect}

One of the most influential demonstrations of sequential control adaptation came from Gratton, Coles, and Donchin (1992), using the Eriksen flanker task. Participants had to identify a letter in the center of a letter string that was either identical to its surrounding flanker letters (compatible trials; e.g., "HHHHH," "SSSSS") or different from them (e.g., "HHSHH," "SSHSS"). The flanker interference is the robustly observed effect of higher reaction times and error rates for incompatible than for compatible trials. Results revealed the so-called Gratton effect, whereby this flanker interference on incompatible trials was smaller after a preceding incompatible trial than after a preceding compatible trial. ${ }^{1}$ Since then, comparable sequential-adaptation effects have been reported for other response interference tasks, such as the Simon

\section{Corresponding Author:}

Gesine Dreisbach, Department of Experimental Psychology, University of Regensburg, Universitätsstraße 31, 93053 Regensburg, Germany E-mail: gesine.dreisbach@psychologie.uni-regensburg.de 


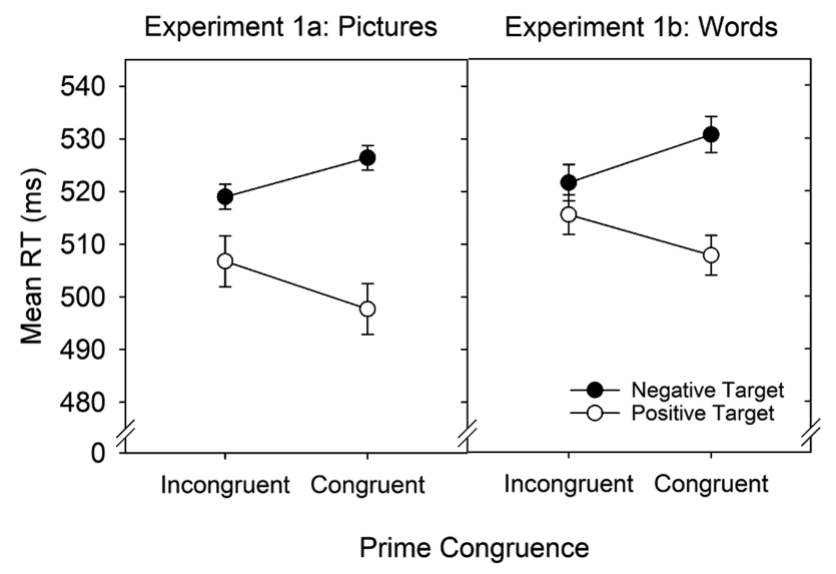

Fig. 1. Results from Dreisbach and Fischer (2012a) demonstrating faster categorizations of negative and slower categorizations of positive target pictures and words following incongruent compared to congruent Stroop primes. RT = reaction time. Adapted from "Conflicts as Aversive Signals," by G. Dreisbach and R. Fischer, 2012, Brain and Cognition, 78, p. 96. Copyright 2012 by Elsevier. Adapted with permission.

task (Stürmer, Leuthold, Soetens, Schröter, \& Sommer, 2002), the Stroop task (Kerns et al., 2004), and even dualtask contexts (Fischer, Gottschalk, \& Dreisbach, 2014).

About 10 years later, Botvinick and colleagues (2001) provided a formal (computational) model explaining these sequential control adjustments. Basically, they suggested a conflict-monitoring module (located in the anterior cingulate cortex, ACC) that gets activated by the concurrent activation of conflicting response alternatives. This conflict signal is then sent to a control unit (e.g., the dorsolateral prefrontal cortex, DLPFC), which in turn sends biasing signals back to the input units, consequently reducing the conflict in the upcoming conflict trial. The beauty of the model (and its success) lies in its independence of an omniscient homunculus: The cognitive system does not have to know the correct answer; it simply detects the difficulties due to incompatible response activations and increases cognitive control. This rather deterministic approach, however, does not explain what exactly it is that motivates this sequential control adaptation.

\section{What Is It That Motivates Conflict Adaptation?}

In the original conflict model by Botvinick and colleagues, the conflict is computed over the output nodes and, after reaching a certain threshold, automatically signals the need for cognitive control to the task unit. The question we ask is, why should the concurrent activation of competing responses motivate the investment of cognitive resources when it is not even clear what the next trial will bring? Even though such a conflict-control loop seems adaptive, we assume that conflict occurrence alone cannot be the sole trigger for conflict adaptation because conflict (measureable response interference) does not always result in conflict adaptation (Desender, Van Opstal, \& Van den Bussche, 2014). We therefore suggest an alternative framework that emphasizes the aversive nature of conflicts as the driving force (cf. Botvinick, 2007). Specifically, we argue that (a) conflicts are inherently aversive and that (b) this aversive conflict experience triggers processes aimed at down-regulating the aversive conflict signal. From there, we speculate that conflict adaptation, so far taken as an indication of sequential action regulation, might actually represent an instantiation of affect regulation. To substantiate our arguments, we will now review recent evidence from our labs.

\section{Conflicts are aversive signals}

The first indirect evidence for the aversive nature of conflict stemmed from a study suggesting that random gain cues (random cues between trials that signaled rewards that were not contingent on the preceding performance) between conflict trials counteract the aversive conflict signal and consequently eliminate conflict adaptation (van Steenbergen, Band, \& Hommel, 2009). In two studies, we provided the first direct evidence for the aversive nature of response conflicts using the affective priming paradigm (Fazio, Sanbonmatsu, Powell, \& Kardes, 1986). The logic behind this paradigm is that the affective valence of primes presented shortly before unambiguous positive or negative targets modulates the affective evaluation of those targets accordingly. In the first study, we therefore presented congruent and incongruent Stroop color words (e.g., "RED" printed in red vs. green) as primes, which were followed by unambiguous positive and negative stimuli (Experiment 1a: positive and negative pictures; Experiment 1b: positive and negative words). Participants then gave speeded affective evaluations of the targets. In both experiments, we found an interaction of prime congruence and target valence: Responses to negative targets were faster after incongruent than after congruent primes, and responses to positive targets were slower after incongruent than after congruent primes (Dreisbach \& Fischer, 2012a; see Fig. 1). In a second study, we showed that conflict primes not only ease the evaluation of negative targets, they also increase the number of negative judgments of neutral targets (Fritz \& Dreisbach, 2013). Taken together, these studies thus provided converging evidence that conflict stimuli are aversive (see also Schouppe et al., 2015). 


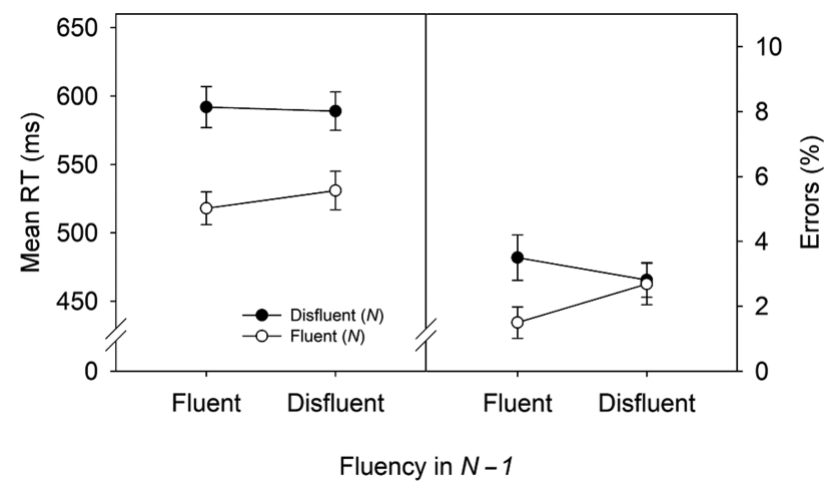

Fig. 2. Results from Experiment 3 of Dreisbach and Fischer (2011). Participants had to categorize written number stimuli ("one," "two," "three," "four," "six," "seven," "eight," "nine") according to magnitude (smaller vs. bigger than 5). Number words occurred in either a fluent or a disfluent font. The figure shows reaction times (left panel) and error rates (right panel) as a function of fluency in trial $N-1$ and fluency in trial $N$. RT = reaction time. Adapted from "If It's Hard to Read... Try Harder!' Processing Fluency as a Signal for Effort Adjustments," by G. Dreisbach and R. Fischer, 2011, Psychological Research, 75, p. 381. Copyright 2011 by Springer. Adapted with permission.

\section{Aversive signals trigger sequential adaptation even in the absence of conflict}

It might seem counterintuitive that an aversive signal should have any motivational function other than avoidance. However, exactly this kind of motivation by frustration has proven to be a very strong motivator under conditions of partial reinforcement schedules (e.g., Amsel, 1992). The parallel we see here is that an aversive signal (e.g., the lack of reward) can indeed be a strong force for increased effort investment. Now, if it is the aversive nature of conflicts that initially motivates sequential control adaptations, aversive stimulus features beyond response conflicts should also trigger sequential processing adjustments. In three experiments, we implemented the affective valence of fluency of processing as an aversive stimulus feature (Winkielman, Schwarz, Fazendeiro, \& Reber, 2003). Fluency of processing describes the experienced ease of perceptual and/or motor processing-as an example, imagine the annoying experience of reading a bad copy from a printer that is running out of toner. Accordingly, we manipulated the perceptual fluency by varying the readability of written numbers. We predicted a fluency effect (faster reaction times for fluent numbers) and a sequential modulation of the fluency effect (i.e., a smaller fluency effect following disfluent stimuli). Results fully confirmed our hypotheses: Aversive (disfluent) stimuli triggered sequential processing adjustments in the absence of conflict (Dreisbach \& Fischer, 2011; see Fig. 2).

\section{Aversive conflict signals trigger affective counter-regulation}

In another study, we manipulated the presentation duration of Stroop primes that were always followed by neutral target stimuli. Participants provided speeded affective evaluations of the target stimuli. Results replicated increased negative judgments for neutral targets after incongruent primes, but only with the usual short (i.e., 200- and 400-millisecond) prime presentation times. Increasing the prime presentation duration to 800 milliseconds, however, significantly reversed the effect: Neutral targets were eventually evaluated even more positively after incongruent Stroop primes than after congruent Stroop primes (Fritz \& Dreisbach, 2015; see Fig. 3).

With increasing conflict persistence, the aversive signal thus seems to initiate processes of affective counterregulation to reduce the aversive conflict experience (see Rothermund, 2011). This also explains why aversive signals that are not part of the conflict stimulus but are presented in between conflict trials eliminate conflict adaptation, as they draw on resources that are then no longer available for adaptation (e.g., Padmala, Bauer, \& Pessoa, 2011). Another closely related finding is that conflict-priming effects reverse when participants are asked to respond to the conflict prime first and to evaluate the affective target afterward (Schouppe et al., 2015). This effect was predicted on the basis of the idea that conflict resolution is rewarding (see also Braem, Verguts, Roggeman, \& Notebaert, 2012; Dreisbach \& Fischer, $2012 \mathrm{~b}$ ) and therefore associated with positive affect. Both studies thus converged on the idea that conflicts motivate processes to end the aversive conflict experience either by solving the conflict or-if the conflict persists-by affective counter-regulation.

Taken together, the reviewed evidence provides strong support for the idea that conflict stimuli are inherently aversive, that sequential control adaptation does not rely on the presence of response conflict (but, rather, on aversive signals), and that conflict stimuli motivate affective counter-regulation.

\section{What Is the Function of the Aversive Conflict Signal?}

The aversive conflict signal might be a mere byproduct of the reduced fluency of processing of conflict stimuli. Alternatively - and this is the explanation we favor-it is the aversive signal, and not the conflict proper, that initially motivates sequential conflict adjustments. In fact, the results from one recent study (Desender et al., 2014) might be interpreted in support of this assumption. There, sequential conflict adaptation was found only after 


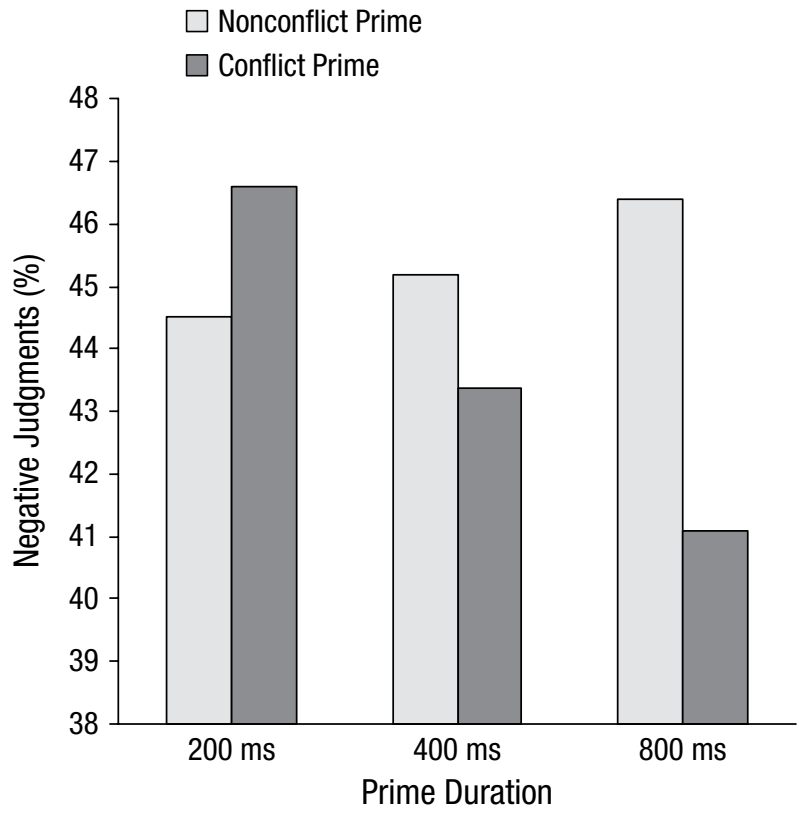

Fig. 3. Results from Fritz and Dreisbach (2015) demonstrating the decreasing frequency of negative target evaluations with increasing conflict-prime presentation duration (200 milliseconds, 400 milliseconds, 800 milliseconds) and a constant prime-target interval of 800 milliseconds. Adapted from "The Time Course of the Aversive Conflict Signal," by J. Fritz and G. Dreisbach, 2015, Experimental Psychology, 62, p. 36. Copyright 2015 by Hogrefe Publishing. Adapted with permission.

conflict trials that were also subjectively experienced as such, whereas the response interference itself (the performance difference between congruent and incongruent trials) was completely unaffected by subjective experience. $^{2}$ This shows that a response conflict can be only a necessary but not a sufficient precondition for sequential conflict adaptation to occur. The original conflict model (Botvinick et al., 2001), however, does not leave much room to explain this observed dissociation between conflict detection and subjective conflict experience. Interestingly, there is one brain structure that-in most studies-is activated jointly with the conflict-detector ACC-namely, the anterior insular cortex (AIC; Craig, 2009). This structure might be sensitive to the subjective conflict experience, just as it has been proven to be sensitive to all sorts of subjective bodily experiences, including interoception, body sensations, and emotional awareness (Craig, 2009). This division of labor might thus provide the neuropsychological basis for the assumed interplay between subjective conflict experience (AIC), conflict detection (ACC), and conflict adaptation (DLPFC). ${ }^{3}$ As a working hypothesis, we therefore suggest that it is the subjective (and presumably aversive) conflict experience that motivates sequential control adaptations, whereas the specific conflict stimulus informs about the specific measures needed to solve the conflict.

\section{Implications}

The idea that the aversive nature of conflicts motivates sequential conflict adaptation opens new perspectives for the investigation of cognitive-control adjustments, especially when it comes to intra- and interindividual differences. One prediction would be that participants with known difficulties experiencing bodily sensations (cf. Damasio, 1996) might also show a reduced adaptation to conflict. Conversely, participants with a sustained or transiently enhanced sensitivity for negative signals, such as participants who score high on trait anxiety (Osinsky, Alexander, Gebhardt, \& Hennig, 2010) or participants in a negative (mood-congruent) state (van Steenbergen, Band, \& Hommel, 2010; van Steenbergen, Booij, Band, Hommel, \& van der Does, 2012), might show an even stronger adaptation to the aversive conflict signal. From the perspective taken here, difficulties in conflict adaptation might thus be ascribed to either an emotional or a cognitive deficit (cf. de Galan, Sellaro, Colzato, \& Hommel, 2014). Differences in the amount of individual conflict adaptation might then be ascribed either to the individual sensitivity to experience the aversive conflict signal or to the individual ability to use the conflict signal for control adaptations. At this point, however, it is hard to decide between these two possibilities. One possible way to resolve this might be to measure the individual conflict sensitivity via conflict priming (Dreisbach \& Fischer, 2012a; Fritz \& Dreisbach, 2013, 2015) as one predictor of conflict adaptation.

\section{Conclusion}

On the basis of the reviewed evidence, we have argued that it is the aversive signal of conflicts that motivates sequential control adaptation, thereby placing conflicttriggered action regulation into the broader context of affect regulation. Highlighting the motivational and affective components in the conflict-triggered adjustments of cognitive control should offer fertile ground and new perspectives for investigating and understanding intraand interindividual differences in the regulation of cognitive control. On a broader level, such an approach will further our understanding of the fascinating interplay of motivational, affective, and cognitive processes in flexible human action control.

\section{Recommended Reading}

Botvinick, M. (2007). (See References). Provides an integrative view of the role of the anterior cingulate cortex in cognitive control.

Dreisbach, G. \& Fischer, R. (in press). Conflicts as aversive signals: Motivation for control adaptation in the service of affect regulation. In Todd S. Braver (Ed.), Motivation and 
cognitive control. New York, NY: Psychology Press. Places the ideas presented here in a broader context.

\section{Acknowledgments}

Part of the research presented here was sponsored by Deutsche Forschungsgemeinschaft Grant DR 392/6-1 to G. Dreisbach.

\section{Declaration of Conflicting Interests}

The authors declared that they had no conflicts of interest with respect to their authorship or the publication of this article.

\section{Notes}

1. The technical term used in the field is sequential conflict adaptation; a more generic term is sequential control adaptation or sequential control adjustment. All terms describe the observation that control requirements experienced in trial $N$ modulate the invested amount of control in trial $N+1$.

2. Conflict (vs. no conflict) was induced by a mismatch (vs. match) between a subliminally presented arrow and an arrow target. Participants were asked after each trial whether they had "felt" a conflict or not. Note that this feeling was independent of the conscious conflict experience.

3. There have been further ideas of division of labor associated with subregions along the dorsal versus rostral part of the ACC (e.g., Bush, Luu, \& Posner, 2000; van Steenbergen, Band, Hommel, Rombouts, \& Nieuwenhuis, 2014). This segregationist view, however, has been criticized (for a meta-analysis, see Shackman et al., 2011).

\section{References}

Amsel, A. (1992). Frustration theory: An analysis of dispositional learning and memory. Cambridge, UK: Cambridge University Press.

Botvinick, M. (2007). Conflict monitoring and decision making: Reconciling two perspectives on anterior cingulate function. Cognitive, Affective, \& Behavioral Neuroscience, 7 , 356-366.

Botvinick, M., Braver, T. S., Barch, D. M., Carter, C. S., \& Cohen, J. D. (2001). Conflict monitoring and cognitive control. Psychological Review, 108, 624-652.

Braem, S., Verguts, T., Roggeman, C., \& Notebaert, W. (2012). Reward modulates adaptations to conflict. Cognition, 125, 324-332.

Bush, G., Luu, P., \& Posner, M. I. (2000). Cognitive and emotional influences in anterior cingulate cortex. Trends in Cognitive Sciences, 4, 215-222.

Craig, A. D. (2009). How do you feel-now? The anterior insula and human awareness. Nature Reviews Neuroscience, 10, 59-70.

Damasio, A. R. (1996). The somatic marker hypothesis and the possible functions of the prefrontal cortex. Proceedings of the Royal Society B: Biological Sciences, 351, 1413-1420.

de Galan, M., Sellaro, R., Colzato, L., \& Hommel, B. (2014). Conflict adaptation is predicted by the cognitive, but not the affective alexithymia dimension. Frontiers in Psychology, 5, Article 768. Retrieved from http://journal.frontiersin.org/ article/10.3389/fpsyg.2014.00768/full
Desender, K., Van Opstal, F., \& Van den Bussche, E. (2014). Feeling the conflict: The crucial role of conflict experience in adaptation. Psychological Science, 25, 675-683. doi:10.1177/0956797613511468

Dreisbach, G., \& Fischer, R. (2011). If it's hard to read . . . try harder! Processing fluency as signal for effort adjustments. Psychological Research, 75, 376-383. doi:10.1007/s00426010-0319-y

Dreisbach, G., \& Fischer, R. (2012a). Conflicts as aversive signals. Brain and Cognition, 78, 94-98.

Dreisbach, G., \& Fischer, R. (2012b). The role of affect and reward in the conflict-triggered adjustment of cognitive control. Frontiers in Human Neuroscience, 6, Article 342. Retrieved from http://journal.frontiersin.org/article/ 10.3389/fnhum.2012.00342/full

Egner, T. (2007). Congruency sequence effects and cognitive control. Cognitive, Affective, \& Behavioral Neuroscience, 7 , 380-390.

Fazio, R. H., Sanbonmatsu, D. M., Powell, M. C., \& Kardes, F. R. (1986). On the automatic activation of attitudes. Journal of Personality and Social Psychology, 50, 229-238.

Fischer, R., Gottschalk, C., \& Dreisbach, G. (2014). Contextsensitive adjustment of cognitive control in dual-task performance. Journal of Experimental Psychology: Learning, Memory, and Cognition, 40, 399-416. doi:10.1037/a0034310

Fritz, J., \& Dreisbach, G. (2013). Conflicts as aversive signals: Conflict priming increases negative judgments for neutral stimuli. Cognitive, Affective, \& Behavioral Neuroscience, 13, 311-317. doi:10.3758/s13415-012-0147-1

Fritz, J., \& Dreisbach, G. (2015). The time course of the aversive conflict signal. Experimental Psychology, 62, 30-39.

Gratton, G., Coles, M. G., \& Donchin, E. (1992). Optimizing the use of information: Strategic control of activation of responses. Journal of Experimental Psychology: General, 121, 480-506.

Kerns, J. G., Cohen, J. D., MacDonald, A. W., III, Cho, R. Y., Stenger, V. A., \& Carter, C. S. (2004). Anterior cingulate conflict monitoring and adjustments in control. Science, 303, 1023-1026.

Miller, E. K., \& Cohen, J. D. (2001). An integrative theory of prefrontal cortex function. Annual Review: Neuroscience, 24, 167-202.

Osinsky, R., Alexander, N., Gebhardt, H., \& Hennig, J. (2010). Trait anxiety and dynamic adjustments in conflict processing. Cognitive, Affective, \& Behavioral Neuroscience, 10, 372-381.

Padmala, S., Bauer, A., \& Pessoa, L. (2011). Negative emotion impairs conflict-driven executive control. Frontiers in Psychology, 2, Article 192. Retrieved from http://journal .frontiersin.org/article/10.3389/fpsyg.2011.00192/full

Rothermund, K. (2011). Counter-regulation and control-dependency: Affective processing biases in the service of action regulation. Social Psychology, 42, 55-66.

Schouppe, N., Braem, S., De Houwer, J., Silvetti, M., Verguts, T., Ridderinkhof, K. R., \& Notebaert, W. (2015). No pain, no gain: The affective valence of congruency conditions changes following a successful response. Cognitive, Affective, \& Behavioral Neuroscience, 15, 251-261.

Shackman, A. J., Salomons, T. V., Slagter, H. A., Fox, A. S., Winter, J. J., \& Davidson, R. J. (2011). The integration of 
negative affect, pain, and cognitive control in the cingulate cortex. Nature Reviews Neuroscience, 12, 154-167.

Stürmer, B., Leuthold, H., Soetens, E., Schröter, H., \& Sommer, W. (2002). Control over location-based response activation in the Simon task: Behavioral and electrophysiological evidence. Journal of Experimental Psychology: Human Perception and Performance, 28, 1345-1363.

van Steenbergen, H., Band, G. P., \& Hommel, B. (2009). Reward counteracts conflict adaptation. Evidence for a role of affect in executive control. Psychological Science, 20, 1473-1477.

van Steenbergen, H., Band, G. P., \& Hommel, B. (2010). In the mood for adaptation: How affect regulates conflict-driven control. Psychological Science, 21, 1629-1634. van Steenbergen, H., Band, G. P., Hommel, B., Rombouts, S. A., \& Nieuwenhuis, S. (2014). Hedonic hotspots regulate cingulate-driven adaptation to cognitive demands. Cerebral Cortex. Advance online publication. doi:10.1093/cercor/bht416

van Steenbergen, H., Booij, L., Band, G. P., Hommel, B., \& van der Does, A. J. (2012). Affective regulation of cognitive-control adjustments in remitted depressive patients after acute tryptophan depletion. Cognitive, Affective, \& Behavioral Neuroscience, 12, 280-286. doi:10.3758/s13415-011-0078-2

Winkielman, P., Schwarz, N., Fazendeiro, T. A., \& Reber, R. (2003). The hedonic marking of processing fluency: Implications for evaluative judgements. In J. Musch \& K. C. Klauer (Eds.), The psychology of evaluation: Affective processes in cognition and emotion (pp. 189-217). Mahwah, NJ: Erlbaum. 